\title{
Initiation of a new connection
}

The molecular mechanisms that regulate synapse formation are not well understood. Eroglu et al. show that thrombospondin (TSP) binding to the neuronal $\alpha 2 \delta$ - 1 receptor initiates the formation of new synapses in vitro and in vivo and that the anti-epileptic and analgesic drug gabapentin (GBP) might function by inhibiting this interaction and hence synaptogenesis.

TSPs are multi-domain, extracellular matrix proteins that are secreted by astrocytes when synapse formation takes place during development.

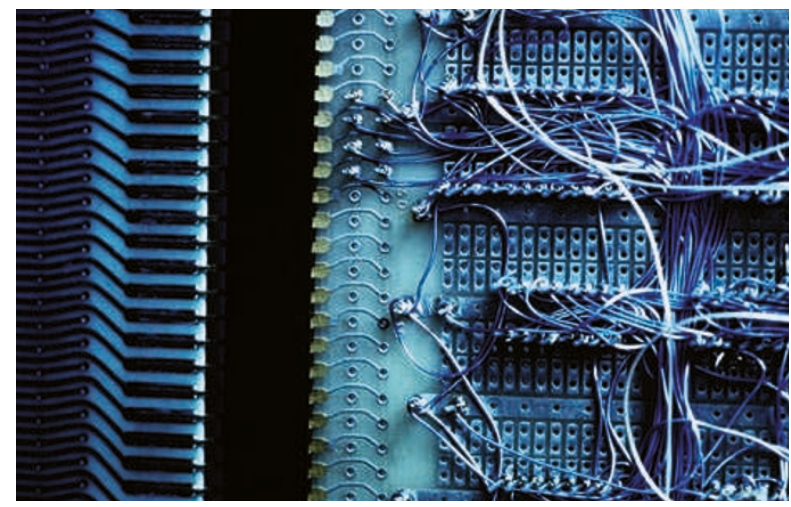

The authors identified the cell surface protein $\alpha 2 \delta$ - 1 as the neuronal TSP receptor that mediates synaptogenesis. Overexpression and knockdown of $\alpha 2 \delta$ - 1 in cultured retinal ganglion cells (RGCs) respectively increased and reduced the number of synapses in the

presence of TSPs. In addition, overexpression of $\alpha 2 \delta-1$ in CNS neurons enhanced synapse formation in vivo, indicating that binding of TSPs to a $2 \delta$ - 1 promotes synaptogenesis in vitro and in vivo.

The authors showed that GBP, a drug that is known to bind to $\alpha 2 \delta$ - 1 with high affinity, inhibited TSP-induced synapse formation in cultured RGCs. Furthermore, the number of synapses was significantly decreased in around $50 \%$ of mice injected neonatally with GBP.

The authors also tested whether TSP-induced synapse formation is involved in injury-induced modification of circuits in the barrel cortex. Mice that were injected with GBP after unilateral removal of some of their whiskers lost the normal topological organization of the barrel cortex, similar to the barrel cortex phenotype found in $T s p 1^{-1-} T s p 2^{-1-}$ mice. These results suggest a role for the TSP- $\alpha 2 \delta$ - 1 interaction in (re)wiring the barrel cortex post-injury and during development. In these studies GBP affected only $30-50 \%$ of mice, an observation that requires further investigation.

In addition to identifying $\alpha 2 \delta-1$ as a receptor that mediates synaptogenesis through the binding of TSPs, this study implies a role for astrocyte-released TSP in the pathophysiology of neuropathic pain and epilepsy, in which aberrant excitatory synaptogenesis is thought to be involved. How GBP mediates its therapeutic function is not very clear, and these results suggest that it could be through limiting new synapse formation.

Claudia Wiedemann

ORIGINAL RESEARCH PAPER Eroglu, Ç. et al.

Gabapentin receptor $\alpha 2 \delta-1$ is a neuronal thrombospondin receptor responsible for excitatory CNS synaptogenesis. Cell 139 . 380-392 (2009) 